\title{
Task based segmentation in personalising e-government services
}

\author{
Sohail Sarwar \\ University of Sunderland \\ Department of Computing, \\ Engineering \& Technology \\ Sunderland,UK \\ sohailsarwarkhan@hotmail.com
}

\author{
Lynne Hall \\ University of Sunderland \\ Department of Computing, \\ Engineering \& Technology \\ Sunderland, UK \\ Lynne.Hall@sunderland.ac.uk
}

\begin{abstract}
eGovernment services whilst functional are frequently underused with a recognised need to improve the user experience. Segmenting users can provide tremendous opportunities to effectively communicate with users and assess user needs for a product or service design. This is equally crucial in designing citizen-centred personalised e-government services, where information needs to be tailored based on user segments sharing common attributes. Commonly used strategies to define user segments include segmentation based on demographics such as age, gender, education etc. or geographic information. Focusing on all facets of users is time consuming and difficult to achieve with the huge, diverse user populations of e-government services. As an alternative to user segmentation based on user characteristics, this paper proposes a task based user segmentation approach with an emphasis on the personalisation of task fulfilment, with user segments derived from the tasks being designed.
\end{abstract}

e-government, personalisation, task analysis, segmentation

\section{INTRODUCTION}

eGovernment is a means to provide services and information online to facilitate their access to citizens and other stakeholders. However, despite extensive Internet use across Europe and high availability of e-government services, recent statistical surveys (Office for National Statistics, 2013; Seybert and Reincake, 2013) have revealed that e-government services are underused and lagging behind other sectors.

The European Commission's Digital Economy and Society Index (DESI) 2017 found that, although the number of e-government users in the UK were slightly raised from $34 \%$ in 2015 to $36 \%$ in 2016, the use of e-government services remains low (European Commission, 2017). The report highlighted that, for the UK, the availability of prefilled forms contributes to the quality, scored only 17 out of 100 further impacted by online service completion scored at 77 out of 100 .

Whilst there are a number of quality factors for online public services, the key factors for citizens are to enable them easily and quickly find the required information and/or solution and to apply it. However, a demand side citizens survey of 28,000 internet using EU citizens across 32 participating countries showed that less than half $(47 \%)$ of the $46 \%$ of EU citizens who used various e-government services, fully got what they wanted from the service. $46 \%$ partially received what they were looking for and 5\% did not get what they wanted at all (European Commission, 2013). This report recognised that public e-services are falling behind commercial services and that government services must be designed and delivered in a citizen-centric manner.

Citizen-centric services need to go beyond good design and navigation. Instead, like the services they are replacing, they should provide users with tailored information and a personalised experience. eGovernment personalisation has been limited, challenged by a vast range of users and services and it remains unclear how personalisation should be provided for citizens (Al-Hassan, 2014, p.2).This paper outlines an approach to the design of a service delivery framework aiming 
to provide e-government services with interaction and information tailored to the user. We outline the task-based segmentation approach that we have developed and applied, demonstrating its use through the example of Garden Waste Collection Service (GWCS), one of the most commonly requested e-government services in the UK.

\section{USER CENTRED DESIGN APPROACHE TO PERSONALISATION}

Personalisation provides a set of techniques that are used to provide a better user experience by tailoring the information and services according to user needs. Personalisation techniques, no doubt, provide better adaptive features to the users but does personalisation provide real value to the end user and where does this fit in the overall design process? This study has found two UCD approaches to personalisation (Kramer et al., 2000; Van Velson et al., 2010). Both of these UCD approaches focus on the individuals and their user experience. With such personalisation, the goal is typically to improve the relationship with the user over time. However, unlike e-commerce or social networking sites designed for frequent users, a majority of the user group does not use e-government services regularly. Thus, a key challenge for e-government is to achieve a personalized service where users are typically casual using services infrequently and often only once. In addition, these approaches could not specify a method to segment users to evaluate adaptive facets of a personalised system.

The next section looks into detail how to personalise system by task rather than user.

\subsection{Task-based user segmentation for e- government personalisation}

There is a perceived need for user data to be as complete as possible and to gather sufficient user information so that no user segments are excluded (Young, 2005). With the potential for big data, it may become possible to hyper-customise the user experience. However, this view of an exhaustive list of user segments based on demographics, geographic location, attitude etc. is beyond that needed for e-government services. Instead, a key challenge for e-government services is that users are typically casual using services infrequently and often only once.

Unlike existing UCD approaches of Kramer et al. (2000) and Van Velson et al. (2010), our focus, after the initial stage of user familiarisation, is on the task rather than the user per se. This task-based user segmentation approach to personalize egovernment services follows similar steps to earlier approaches but with techniques focused on task fulfilment as the basis for personalisation.

STEP 1: User Research: is conducted to gain an overall understanding of the diverse user group Simple target user segments such as families, retired people, drivers, etc. are identified and targeted Personas are built to understand user goals, desires, behaviour and pain points in using egovernment services. This provides an early focus on needs, expectations and uncovering the issues in current provision.

STEP 2: Service selection: we propose an incremental approach to personalise services where the most important and/or most used set of services is chosen to personalise. This may include services critical for the business or commonly used services. Where e-government services, are in use, website analytics should be used to prioritise commonly searched for and/or abandoned services.

STEP 3: Deriving user segments from task analysis : of current user activities, using the selected services to get in depth understanding of how users access the existing services to achieve their goals. Extended Hierarchical Task Analysis (HTA) is used for task decomposition with the user segments derived from task based attributes. Within an extended HTA (as detailed in Figure 1 below) the decision points are represented by diamond symbols. Tasks in the form of user actions/activities are represented by rectangle symbols. The decision points provide useful information, based on which users can be segmented and key user segmentation points are represented by encircling the diamond symbols with green circles.

STEP 4: Prototyping: lo-fi and mid-tech prototypes are designed to enable rapid, easy task fulfilment. They are used to gather feedback as various personalisation features and system adaptations are incorporated and integrated into a high fidelity prototype.

STEP 5: Evaluation: the service prototype is evaluated by users using questionnaires, interviews, focus group discussions, think aloud, expert reviews, etc. Usability, perceived usefulness, user satisfaction, appropriateness of adaptation etc. (Van Velson, 2011) are evaluated at this stage with related refinements undertaken. 
STEP 6: Roll out: service implemented, tested and deployed. The deployed system should allow users to submit their feedback.

In this paper, we are focusing on STEP 3, with the following study outlining how we applied a broadbrush approach to personalisation based on user segmentation through tasks.

\section{CASE STUDY - GARDEN WASTE}

This research focuses on the task analysis of Garden Waste Collection Service (GWCS) provided by Durham County Council, a local government authority in the UK. GWCS is one of the most frequently searched for services, which offers a fortnightly garden waste collection for more

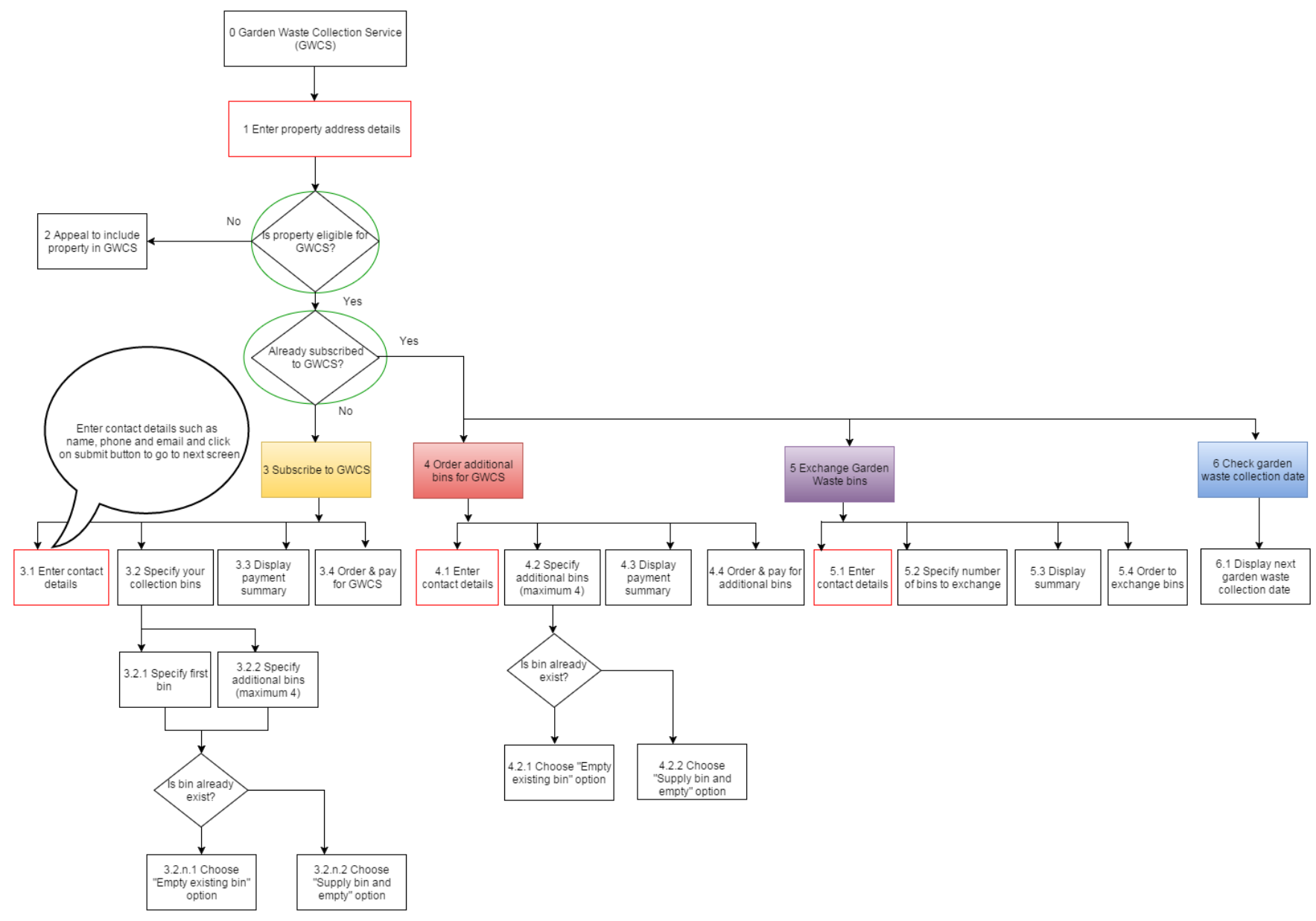

of additional bins, exchange bins (a scheme to provide larger bins) and to check garden waste collection dates.

The various decision points represented by diamond symbols in Figure 1 provide us with an approach to identify various user segments for which services could be personalised. The decision: "Is property eligible for GWCS" identifies three user segments i) Users with properties eligible for GWCS, ii) Users with properties not eligible for GWCS and a third segment iii) users appealing their current eligibility status. Further decision points indicate that some of these segments can be further divided into sub segments. For example eligible properties might have already been registered for the garden waste collection, speeding up subscription.

Through allocating users to a segment, they are then

Figure 1: Hierarchical Task Analysis and task flow diagram of Garden Waste Collection Service

than 190,000 properties across the County and charge a fee for this service.

Hierarchical Task Analysis of the existing system was carried out and the flow of information was analysed throughout the process as shown in Figure 1. A number of specialised services related to Garden Waste are offered, including the collection only presented with the tasks under the segment branch. Thus, the service rather than being generic and catch-all is tailored to the user with this task restriction providing the basis of service personalization. Once tasks are fulfilled, for example "Subscribe to GWCS", this becomes superfluous and is not displayed unless the subscription is 
cancelled or lapses. Similarly order additional bins, exchange bins, check collection dates, etc. tasks are displayed when the user property has already been registered for the GWCS. Along with other tasks, user profiling tasks are a key element of the personalised version. User profiles contain information about the individual users themselves. Here, we propose that the

profiles are extended to include attributes that represent the segment(s) that the user is in. For example, using a rule based approach (Borkowski, 2011), a Boolean attribute "Garden waste eligibility" could be added to user profile to represent if a user property is eligible for GWCS.

Segmenting users as those living in a property eligible for GWCS or not, influences the design of the personalised dashboard that will allow citizens to access e-government services. For example, those who have subscribed to the GWCS will have collection dates and other garden waste collection tasks. For those who do not have eligibility for GWCS, this service would not be displayed freeing up screen space for other, more useful information. Through segmenting at each decision point, users are provided with tailored information. However, this personalisation is not aimed at the individual users but rather to the tasks that the user segment aims to complete.

\section{CONCLUSIONS}

This paper proposes an approach for personalising e-government services through segmenting users based on task fulfilment. This approach enables, personalisation with the experience tailored to the user through incorporating or removing tasks and services dependant on the user segment. This relatively simplistic approach enables designs to be meaningfully personalised at a relatively low cost to the e-government service provider. With the key goal for e-government services to provide appropriate, rapid, easy-to-use services, this task based user segmentation approach offers the potential to improve the user experience of $e$ government services. Currently, our work is testing the validity of this approach. Further work is required to design a personalised prototype and evaluate the prototype by the user segments.

\section{REFERENCES}

Al-Hassan, M. (2014), Semantic-Enhanced Hybrid Recommender Systems for Personalised EGovernment Services, PhD thesis, University of Technology, Sydney [online], Available at: https://opus.lib.uts.edu.au/research/handle/1045 3/30504 (Accessed: 01 November 2016).

Borkowski, G. (2011), "Personalization fundamentals (part 2): Rules-based personalization", Oracle Blogs, 26 September. Available

at:

https://blogs.oracle.com/atgstrategy/entry/person alization fundamentals part 2 rules (Accessed: 10 October 2015).

European Commission (2013), Public Services Online 'Digital by Default or by Detour?', Available at:

market/sites/digital-

https://ec.europa.eu/digital-single-

agenda/files/eGov\%20Benchmark\%202012\%20i nsight\%20report\%20published\%20version\%200. 1\%20 0.pdf (Accessed: 4 December 2015).

European Commission (2017), "United Kingdom, Digital Economy and Society Index 2017", Available at: https://ec.europa.eu/digital-singlemarket/en/scoreboard/united-kingdom

(Accessed: 2 February 2017).

Kramer, J., Noronha, S. and Vergo, J. (2000), “A User-Centered Design Approach to Personalization", Communications of the ACM, 43, 8, pp. 44-48.

Office for National Statistics (2013), “Internet Access - Households and Individuals, 2013”, Available at: http://www.ons.gov.uk/ons/dcp171778 322713.p df (Accessed: 2 April 2015).Seybert, H. and Reinecke, P. (2013), "Three quarters of Europeans used the internet in 2013", Available at http://ec.europa.eu/eurostat/statisticsexplained/index.php/Archive:Internet use statist ics - individuals (Accessed: 01 October 2016).

Van Velson, L. S. (2011), User-Centered Design for Personalization, PhD thesis, University of Twente, Enschede [online], Available at: http://doc.utwente.nl/76053/1/thesis L van Vels en.pdf (Accessed: 01 November 2015).

Van Velsen, L., Van der Geest, T. and Steehouder, M. (2010), "The Contribution of Technical Communicators to the User-Centered Design Process of Personalized Systems", Technical Communication, 57, 2, pp. 182-196.

Young, I. (2005), Task-Based Audience Segmentation, Available at: http://adaptivepath.org/ideas/task-basedaudience-segmentation/ (Accessed: 2 November 2016). 